Research Article

\title{
The Bühlmann-Straub Estimation of Claim Means in Random B-F Reserve Model
}

\author{
Zhang Yi, ${ }^{1,2}$ Wen Limin ${ }^{1},{ }^{1}$ and Li Zhilong ${ }^{2}$ \\ ${ }^{1}$ Department of Statistics, Jiangxi Normal University, Nanchang, Jiangxi, China \\ ${ }^{2}$ School of Statistics, Jiangxi University of Finance and Economics, Nanchang, Jiangxi, China
}

Correspondence should be addressed to Wen Limin; wlmjxnu@163.com

Received 22 July 2019; Revised 18 April 2020; Accepted 21 April 2020; Published 7 May 2020

Academic Editor: Bruno G. M. Robert

Copyright (c) 2020 Zhang Yi et al. This is an open access article distributed under the Creative Commons Attribution License, which permits unrestricted use, distribution, and reproduction in any medium, provided the original work is properly cited.

In the B-F reserve model, it is a very critical step to estimate the claim means of the accident year. However, the traditional method uses the prior estimators of the claim means based on the personal experience of actuaries or historical data. This method inevitably carries the subjectivity of the actuary himself. In this paper, a stochastic B-F model is established, and a prior distribution is constructed for the claim means in the accident year. The idea of the credibility theory is used to derive the linear Bayesian estimators of claim means. Finally, the empirical Bayesian method is used to estimate the first two moments of the prior distribution, and the empirical Bayesian estimators of the claim means and the corresponding reserves are derived. The estimators obtained in this paper do not depend on the specific forms of the sample distribution and the prior distribution and can be used directly in practice. In the numerical simulation, our estimates are compared with the traditional B-F estimates and the chain ladder estimates. It is verified that the estimates given in this paper have small mean square error.

\section{Introduction}

The liability reserve of the policy refers to the amount estimated by the actuary to pay the insurance claims due in the future. The insurance company must ensure that it has sufficient capital reserves so that it has funds to pay future claims. In the non-life insurance business, the reserve is the largest debt account on the balance sheet, which is directly related to the level of the insurance company's profit index and the solvency ability. Due to the influence of many complex factors, the assessment of non-life insurance business reserves has been a hot and difficult issue in actuarial theory and applications.

Among the many liability reserve models, the distribution-free chain ladder (CL) and Bornhuetter-Ferguson (B-F) method are still the most frequently used claims reserving methods in practice. Refer to Mack [1] and Bornhuetter and Ferguson [2]. In order to estimate the amount of reserves, the actuary classified the claim by the year in which the accident occurred. If the vertical axis is considered as the year of occurrence of the accident, and the horizontal axis is taken as the development year after the accident, the claim data will form an upper triangle. In the chain ladder method, it is assumed that each progress year has a stable development factor. Based on this upper triangular data, the claims in each accident year are predicted and the reserve estimates are obtained. This method is called "chain ladder method". Because the chain ladder method is simple to calculate and the calculation result is approximately accurate, it is widely used in the reserve assessment in the non-life insurance company.

Actually, the early chain ladder method has nonrandom form which is called as "nonrandom chain ladder method". However, some researchers began to question this method since it has no relevant theoretical support. Furthermore, it cannot give a good explanation. This question was well resolved in Mack [1]. He gives a stochastic model of the chain method, which not only obtains the same claim reserve estimate as the "nonrandom chain ladder method," but also obtains the error of the estimate. So far, the chain ladder method is still a mainstream approach to reserve estimates. Related studies include Quarg and Mack [3], Hürlimann [4], 
Pešta and Hudecová [5], Riegel [6], Leonardo et al. [7], Boratyńska [8], Wahl et al. [9], and references therein.

Note that, in the chain ladder method, it is assumed that there is a development factor $f_{j}$ in the development year $j$, such that

$$
E\left(C_{i, j+1} \mid C_{i 0}, \ldots, C_{i j}\right)=f_{j} C_{i j}
$$

where $C_{i, j}$ represents the cumulative claims in accident year $i \in\{0,1, \ldots, I\}$ at the end of development year $j \in\{0,1, \ldots, J\}$, and we assume that $J \leq I$. For the B-F method, it is assumed that there are given estimates $\widehat{\mu}_{i}$ of the expected ultimate claim of each accident year $i$ and the development pattern $\gamma_{j}$ in progress year $j$ such that

$$
\begin{array}{r}
E\left(C_{i, j+k} \mid C_{i 0}, \ldots, C_{i j}\right)=C_{i j}+\left(\beta_{j+k}-\beta_{j}\right) \mu_{i} \\
\text { for } j=1,2, \ldots \text { and } j+k \leq J,
\end{array}
$$

with $\beta_{j}=\sum_{k=0}^{j} \gamma_{k}$.

Different from the chain ladder method, the B-F method attempts to obtain a reserve estimate by combining the prior information of the random parameters and the sample information of loss. Due to the complexity of the risk, claims arising from different policies take different values which form a certain probability distribution. Thus, the estimation and statistical inferences of reserve fall into the Bayesian framework. Note that if the prior estimates of claim means are taken as $\widehat{\mu}_{i}=\mu_{i}$ for $i \in\{0,1, \ldots, I\}$, the B-F estimator of the reserve is exactly the same as that of the chain ladder estimator. Therefore, in a sense, the B-F model is a generalization of the ladder method under the Bayesian framework. The reserve model for the B-F method has attracted many researchers in recent years. Related discussion can refer to Verrall [10], Alai et al. [11], Saluza et al. [12], Jochen and Anne [13], and references therein. Obviously, the estimates of claim means $\mu_{i}$ are very crucial in the reserve assessment of the B-F model. A common method is to assume that a prior estimate already exists for $\mu_{i}, i=0,1, \ldots, I$, for example, Gogol [14] and Verrall [10]. It is noted that the prior estimates $\widehat{\mu}_{i}$ for $i \in\{0,1, \ldots, I\}$ are generally determined by the actuary based on the expert opinions or some empirical information which are determined with greater subjectivity. If the prior estimates are appropriately chosen, an accurate estimate of the reserve can be obtained. On the contrary, a greater error is brought into the estimates of reserve if the prior estimates are wrongly chosen.

Therefore, it is very necessary to establish the Bayesian model for $\mu_{i}$ and get appropriate estimates for $\mu_{i}$ by the use of Bayesian statistical inference procedures. In the Bayesian statistical model, it is still very difficult how to choose the appropriate prior distribution for $\mu_{i}$. In this paper, however, we will introduce the credibility theory to estimate the claim means and derive the linear Bayesian estimators of $\mu_{i}$. The estimates obtained depend only on the first two moments of the prior distribution and do not depend on the specific prior distribution. The credibility theory is a premium pricing model by using both sample information and prior information, which is widely used in non-life insurance actuarial science. One can refer to Bühlmann [15], Bühlmann and Gisler [16], Wen et al. [17], Wen et al. [18], and Tsai and $\mathrm{Wu}$ [19] for more detailed introduction of credibility theory. About the research studies of liability reserve by using credibility theory, one can refer to Benktander [20], Hovinen [21], Hesselager and Witting [22], Vylder [23], Gisler and Wüthrich [24], Taylor [25], and so on.

The rest of the paper is arranged as follows. Section 2 gives the assumptions and notations of the model. Section 3 gives the nonhomogeneous and homogeneous credibility estimates of the claim means. In addition, the estimates of structural parameters in credibility factors are derived, and the empirical Bayesian estimates of reserves are discussed. In the fourth section, we give a numerical example to illustrate the calculations of the empirical Bayesian estimators and to compare the credibility estimators obtained in this paper with the traditional chain ladder method and B-F method. Finally, a real example is given to show the results derived in this paper.

\section{Bühlmann-Straub Model for Claim Mean}

In the loss triangle data of the liability reserve, it is assumed that the claims for the different accident years are independent of each other and the cumulative claim for each year $C_{i j}$ satisfies the conditions of the B-F model:

$$
\left\{\begin{array}{l}
E\left(C_{i, 0} \mid \mu\right)=\mu_{i} \beta_{0}, \quad i=0,1,2, \ldots, I, \\
E\left(C_{i, j+k} \mid C_{i, 0}, \ldots, C_{i, j}, \mu\right)=C_{i, j}+\left(\beta_{j+k}-\beta_{j}\right) \mu_{i},
\end{array}\right.
$$

for $j+k \leq J$, where $\mu=\left(\mu_{0}, \ldots, \mu_{I}\right)^{T}$ are claim means for every accident year, and $\beta_{j}=\sum_{k=0}^{j} \gamma_{j}$ with $\gamma_{j}>0$, where the superscript " $T$ " indicates the transpose of the vector. Without loss of generality, one can assume that $\beta_{J}=\sum_{k=0}^{J} \gamma_{k}=1$.

According to the double expectation formula, we get

$$
E\left(C_{i, j} \mid \mu\right)=E\left[E\left(C_{i, j} \mid C_{i, 0}, \ldots, C_{i, j-1}, \mu\right) \mid \mu\right]=\mu_{i} \beta_{j}
$$

Let

$$
X_{i, j}=C_{i, j}-C_{i, j-1}
$$

denote the incremental claims. So the incremental claim $X_{i, j}$ satisfies

$$
E\left(X_{i, j} \mid \mu\right)=\mu_{i} \gamma_{j}
$$

where $\gamma_{j}=\beta_{j}-\beta_{j-1}$.

We assume that the claim means are independent random variables with the same prior distribution. Therefore, we propose the following random B-F model.

Assumption 1. There are function $\sigma^{2}\left(\mu_{i}\right)$ and fixed numbers $w_{i j}$ for $i \in\{0,1,2, \ldots, I\}, j \in\{0,1,2, \ldots, J\}$ such that 


$$
\begin{gathered}
E\left(X_{i, j} \mid \mu\right)=\gamma_{j} \mu_{i}, \\
\operatorname{Var}\left(X_{i, j} \mid \mu\right)=\frac{\sigma^{2}\left(\mu_{i}\right)}{w_{i j}}, \\
\operatorname{Cov}\left(X_{i, j}, X_{i, k}\right)=0 \quad \text { for } j \neq k, \\
k=0,1, \ldots, J .
\end{gathered}
$$

Assumption 2. The $\left\{\mu_{0}, \mu_{1}, \ldots, \mu_{I}\right\}$ are assumed to be independent variables with the same prior distribution $\pi(\mu)$.

Assumption 3. Assume that claims arising from different accident years are independent of each other.

Remark 1. According to Assumptions (1)-(3), this model is similar to the Bühlmann-Straub assumptions in the credibility theory. See, for example, Bühlmann and Gisler [16]. So this model is also called the Bühlmann-Straub model for claim means.

At time $I$, we have the sample information:

$$
D_{I}=\left\{C_{i j}, i+j \leq I, j \leq J\right\}=\left\{X_{i j}, i+j \leq I, j \leq J\right\},
$$

and prior information $\pi(\mu)$. In this paper, our purpose is to estimate $\mu_{i}$ and predict

$$
D_{I}^{c}=\left\{C_{i j}, i+j>I, i \leq I, j \leq J\right\} .
$$

The outstanding loss liabilities for accident year $i$ at time $I$ are given by

$$
\begin{gathered}
R_{i}=C_{i J}-C_{i, I-i}, \\
I-J+1 \leq i \leq I,
\end{gathered}
$$

and the total outstanding loss liabilities of all accident years are given by

$$
R=\sum_{i=I-J+1}^{I} R_{i}
$$

Obviously, if the square loss is used, by minimizing the conditional expectation

$$
E\left[\left(C_{i, J}-g\left(D_{I}\right)\right)^{2} \mid D_{I}\right]
$$

we get the optimal predictors of $C_{i J}$ as

$$
\widehat{C}_{i J}^{*}=C_{i, I-i}+\left(1-\widehat{\beta}_{I-i}\right) E\left(\mu_{i} \mid D_{I}\right) \text {, }
$$

for $i \in\{0,1, \ldots, I\}$, where $\widehat{\beta}_{I-i}$ is the chain ladder estimator of $\beta_{I-i}$, i.e.,

$$
\widehat{\beta}_{j}=\prod_{k=j}^{J-1} \widehat{f}_{k}^{-1}, \quad j=0,1,2, \ldots, J .
$$
by

Here, the estimators of chain ladder factor $f_{j}$ are given

$$
\widehat{f}_{j}=\frac{\sum_{i=0}^{I-j-1} C_{i, j+1}}{\sum_{i=0}^{I-j-1} C_{i, j}}, \quad j=1,2, \ldots, J-1 .
$$

For a detailed introduction to the estimation of development factors, refer to Gisler and Wüthrich [24].

\section{Credibility Estimation of Reserves}

3.1. Nonhomogeneous Credibility Estimation of Claim Mean. According to (14), in order to predict the ultimate loss $C_{i, j}$, the $\widehat{\mu}_{i}=E\left(\mu_{i} \mid D_{I}\right)$ must be solved. In this paper, we introduce the following notations:

$$
\begin{aligned}
E\left(\mu_{i}\right) & =v_{0}, \\
\operatorname{Var}\left(\mu_{i}\right) & =\tau_{0}^{2}, \\
E\left[\sigma^{2}\left(\mu_{i}\right)\right] & =\sigma_{0}^{2},
\end{aligned}
$$

and constrain the estimate of $\mu_{i}$ to the linear function of the sample $D_{I}$. For convenience, it is assumed that $I=J$ in the following. With slight modification, the models can be extended to the case of $I \geq J$.

Under the square loss function, we solve the optimization problem,

$$
\min _{\alpha, \alpha_{s t} \in R} E\left[\left(\mu_{i}-\alpha-\sum_{s=0}^{I} \sum_{t=0}^{I-s} \alpha_{s t} X_{s t}\right)^{2}\right]
$$

and get the following theorem.

Theorem 1. Under Assumptions (1)-(3), by minimizing (18), the nonhomogeneous credibility estimators of $\mu_{i}$ are given by

$$
\widehat{\mu}_{i}^{B S}=Z_{i}^{w} \bar{X}_{i}^{w}+\left(1-Z_{i}^{w}\right) v_{0},
$$

for $i \in\{0,1,2, \ldots, I\}$, where

$$
\bar{X}_{i}^{w}=\frac{1}{w_{i}} \sum_{j=0}^{I-i} w_{i j} \gamma_{j} X_{i j}, Z_{i}^{w}=\frac{\tau_{0}^{2} w_{i}}{\sigma_{0}^{2}+\tau_{0}^{2} w_{i} .},
$$

and $w_{i .}=\sum_{j=0}^{I-i} w_{i j} \gamma_{j}^{2}$

Remark 2. In Theorem 1 , if the weights are taken as $w_{i j}=$ $\left(1 / \gamma_{j^{2}}\right)$ for any $i$ and $j$, we have

$$
\begin{gathered}
E\left(\frac{X_{i, j}}{\gamma_{j}} \mid \mu\right)=\mu_{i}, \\
\operatorname{Var}\left(\frac{X_{i, j}}{\gamma_{j}} \mid \mu\right)=\frac{\sigma^{2}\left(\mu_{i}\right)}{w_{i j} \gamma_{j}^{2}} .
\end{gathered}
$$

In addition, we denote $Y_{i j}=\left(X_{i j} / \gamma_{j}\right)$ and get

$$
\begin{gathered}
E\left(Y_{i, j} \mid \mu\right)=\mu_{i}, \\
\operatorname{Var}\left(Y_{i, j} \mid \mu\right)=\frac{\sigma^{2}\left(\mu_{i}\right)}{w_{i j} \gamma_{j}^{2}},
\end{gathered}
$$

which are exactly the Bühlmann-Straub models for conditional means in credibility theory. In addition, if we take 
$w_{i j}=\left(1 / \gamma_{j}^{2}\right)$, the assumptions are simplified to Bühlmann's model for claims $\left\{Y_{i, j}, \quad i=0,1, \ldots, I, \quad j=0,1, \ldots, J\right\}$. See Bühlmann and Gisler [16] for more detail.

Proposition 1. The $\widehat{\mu}_{i}^{B S}$ is an unbiased estimator of $\mu_{i}$, i.e., $E\left(\widehat{\mu}_{i}^{B S}\right)=E\left(\mu_{i}\right)=v_{0}$, and the mean square error is given by

$$
\operatorname{MSE}\left(\widehat{\mu}_{i}^{B S}\right)=\frac{\tau_{0}^{2} \sigma_{0}^{2}}{\sigma_{0}^{2}+\tau_{0}^{2} \sum_{j=0}^{I-i} w_{i j} \gamma_{j}^{2}} .
$$

By inserting $\widehat{\mu}_{i}^{B S}$ into (14), the estimators of ultimate loss $C_{i, J}$ for $i \in\{0,1, \ldots, I\}$ can be derived, and the estimator of reserve can also be derived, which is stated as the following proposition.

Proposition 2. Under Assumptions (1)-(3), if the estimator of $\mu_{i}$ is taken as $\hat{\mu}_{i}^{B S}$, the estimator of ultimate loss $C_{i J}$ for $i \in\{0,1, \ldots, I\}$ is given by

$$
\widehat{C}_{i J}^{B S}=C_{i, I-i}+\left(1-\widehat{\beta}_{I-i}\right)\left(Z_{i}^{w} \bar{X}_{i}^{w}+\left(1-Z_{i}^{w}\right) v_{0}\right) .
$$

In addition, the estimators of reserve $R_{i}$ and total reserve are given by

$$
\begin{aligned}
& \widehat{R}_{i}^{B S}=\left(1-\widehat{\beta}_{I-i}\right)\left[Z_{i}^{w} \bar{X}_{i}^{w}+\left(1-Z_{i}^{w}\right) v_{0}\right], \\
& \widehat{R}^{B S}=\sum_{i=1}^{I}\left(1-\widehat{\beta}_{I-i}\right)\left[Z_{i}^{w} \bar{X}_{i}^{w}+\left(1-Z_{i}^{w}\right) v_{0}\right],
\end{aligned}
$$

respectively.

3.2. Homogeneous Credibility Estimation. In practice, we note that $\mu_{0}$ in formula (24) is unknown and needs to be estimated by the samples. Similar to the nonhomogeneous credibility estimation method, we define the homogeneous linear function class of $D_{I}$ as

$$
L e(X)=\left\{\widehat{\mu}_{i}=\sum_{s=0}^{I} \sum_{t=0}^{I-s} \alpha_{s t} X_{s t} \text { with } \sum_{s=0}^{I} \sum_{t=0}^{I-s} \gamma_{t} \alpha_{s t}=1\right\} .
$$

and solve the optimal estimator of $\mu_{i}$ in $L e(X)$. We get the following theorem.

Theorem 2. By solving the optimization problem,

$$
\left\{\begin{array}{c}
\min _{\alpha, \alpha_{s t} \in R} E\left[\left(\mu_{i}-\sum_{s=0}^{I} \sum_{t=0}^{I-s} \alpha_{s t} X_{s t}\right)^{2}\right], \\
\sum_{s=0}^{I} \sum_{t=0}^{I-s} \alpha_{s t} \gamma_{t}=1,
\end{array}\right.
$$

we get the optimal homogeneous estimators of $\mu_{i}$ as

$$
\widehat{\mu}_{i}^{\text {whom }}=Z_{i}^{w} \bar{X}_{i}^{w}+\left(1-Z_{i}^{w}\right) \widehat{\nu}_{0}, \quad i=0,1, \ldots, I,
$$

where

$$
\widehat{\nu_{0}}=\frac{\sum_{k=0}^{I} Z_{k}^{w} \bar{X}_{k}^{w}}{\sum_{k=0}^{I} Z_{k}^{w}}
$$

Remark 3. According to the optimal homogeneous credibility estimators of $\mu_{i}$ for $i \in\{0,1, \ldots, I\}$, the homogeneous credibility of the corresponding estimators of ultimate loss $C_{i J}$ is given by

$$
\widehat{C}_{i J}^{\text {whom }}=C_{i, I-i}+\left(1-\widehat{\beta}_{I-i}\right)\left(Z_{i}^{w} \bar{X}_{i}^{w}+\left(1-Z_{i}^{w}\right){\widehat{\nu_{0}}}^{w} .\right.
$$

and the homogeneous credibility estimators of reserve $R_{i}$ are

$$
\widehat{R}_{i}^{\text {whom }}=\left(1-\widehat{\beta}_{I-i}\right)\left[Z_{i}^{w} \bar{X}_{i}^{w}+\left(1-Z_{i}^{w}\right) \widehat{\nu}_{0}\right] \text {, }
$$

for $i \in\{0,1,2, \ldots, I\}$. As a result, the estimator of the total reserve is given by

$$
\widehat{R}^{\text {whom }}=\sum_{i=0}^{I}\left(1-\widehat{\beta}_{I-i}\right)\left[Z_{i}^{w} \bar{X}_{i}^{w}+\left(1-Z_{i}^{w}\right) \widehat{\nu}_{0}\right] .
$$

3.3. The Estimation of Variance Parameters. The credibility estimator of the total reserve is still unable to use in practice because they still contain unknown structural parameters such as $\tau_{0}^{2}$ and $\sigma_{0}^{2}$. In this part, we will give the unbiased estimators of these structural parameters.

Proposition 3. An estimator of $\sigma_{0}^{2}$ is given by

$$
\widehat{\sigma_{0}^{2}}=\frac{1}{I} \sum_{i=0}^{I-1} \frac{1}{I-i} \sum_{j=0}^{I-i} w_{i j}\left(X_{i j}-\gamma_{j} \bar{X}_{i}^{w}\right)^{2}
$$

In addition, the $\widehat{\sigma_{0}^{2}}$ is an unbiased estimator of $\sigma_{0}^{2}$ when $\left\{\gamma_{j}, j=0,1, \ldots, J-1\right\}$ are given.

Proposition 4. An estimator of $\tau_{0}^{2}$ is given by

$$
\widehat{\tau_{0}^{2}}=\frac{w_{. .}}{\left(w_{. .}\right)^{2}-\sum_{i=0}^{I-1}\left(w_{i}\right)^{2}}\left[\sum_{i=0}^{I-1} w_{i \cdot}\left(\bar{X}_{i}^{w}-\overline{\bar{X}}^{w}\right)^{2}-I{\widehat{\sigma_{0}^{2}}}^{2},\right.
$$

where

$$
\overline{\bar{X}}^{w}=\frac{1}{w_{.}} \sum_{i=0}^{I-1} w_{i}{\overline{X_{i}}}^{w}, \quad w_{. .}=\sum_{i=0}^{I-1} w_{i .}, w_{i .}=\sum_{j=0}^{I-i} w_{i j} \gamma_{j}^{2} .
$$

Furthermore, the $\widehat{\tau_{0}^{2}}$ is an unbiased estimator of $\tau_{0}^{2}$ if $\left\{\gamma_{j}, j=0,1, \ldots, J-1\right\}$ are given constants.

Remark 4. If we denote $w_{i j}^{\prime}=\left(1 / \gamma_{j}^{2}\right)$ and take $w_{i j}=w_{i j}^{\prime}$, some symbols degenerate to $w_{i}^{\prime} \cdot=\sum_{j=0}^{I-i} w_{i j}^{\prime} \gamma_{j}^{2}=I-i+1$, $w_{. .}^{\prime}=\sum_{i=0}^{I-1} w_{i .}=(1 / 2) I(I+1)$,

$$
\bar{X}_{i}^{w^{\prime}}=\frac{1}{I-i+1} \sum_{j=0}^{I-i} Y_{i j}, \overline{\bar{X}}^{w^{\prime}}=\frac{2}{I(I+1)} \sum_{i=0}^{I-1}(I-i+1) \bar{Y}_{i} \text {. }
$$

Denote $\bar{Y}_{i}=(1 / I-i+1) \sum_{j=0}^{I-i} Y_{i j}$ and $\overline{\bar{Y}}=\left(2 / \widehat{\widehat{T}}_{(}(I+1)\right)$ $\sum_{i=0}^{I-1}(I-i+1) \bar{Y}_{i}$, and thus, the estimators $\widehat{\sigma_{0}^{2}}$ and $\widehat{\tau_{0}^{2}}$ can be simplified as follows: 


$$
\begin{aligned}
& \widehat{\sigma_{0}^{2}}=\frac{1}{I} \sum_{i=0}^{I-1} \frac{1}{I-i} \sum_{j=0}^{I-i} \frac{1}{\gamma_{j}}\left(Y_{i j}-\bar{Y}_{i}\right)^{2}, \\
& \widehat{\tau_{0}^{2}}=\frac{6}{(3 I+2)(I-1)}\left[\sum_{i=0}^{I-1}(I-i+1)\left(\bar{Y}_{i}-\overline{\bar{Y}}\right)^{2}-I \widehat{\sigma_{0}^{2}}\right],
\end{aligned}
$$

respectively.

In practice, the estimators $\hat{\gamma}_{j}$ for $j \in\{0,1, \ldots, J\}$ are given in terms of the chain ladder method. By inserting $\widehat{\mathcal{V}}_{j}$ into the estimators of $\sigma_{0}^{2}$ and $\tau_{0}^{2}$, then we get the estimators $\sigma_{0}^{2}$ and $\widetilde{\tau_{0}^{2}}$. However, the estimators $\widehat{\sigma_{0}^{2}}$ and $\widehat{\tau_{0}^{2}}$ are no longer unbiased. In the actual data, it is possible that $\widetilde{\tau_{0}^{2}}<0$, we usually take $\widetilde{\tau_{0}^{2}}=\max \left(\widetilde{\tau_{0}^{2}}, 0\right)$ as the ultimate estimator of $\tau_{0}^{2}$.

Finally, by inserting $\left\{\widehat{\gamma}_{j}, j=0,1, \ldots, J-1\right\}$ and $\widetilde{\sigma_{0}^{2}}$ and ${\widetilde{\tau_{0}^{2}}}^{+}$into the estimator of $\nu_{0}$, thus the ultimate estimator of $\nu_{0}$ is given by

$$
\tilde{\nu}_{0}=\frac{\sum_{k=0}^{I} \widehat{Z}_{k}^{w} \bar{X}_{k}^{w}}{\sum_{k=0}^{I} \widehat{Z}_{k}^{w}}
$$

where

$$
\widehat{Z}_{i}^{w}=\frac{{\widetilde{\tau_{0}^{2}}}^{+} \sum_{j=0}^{I-i} w_{i j} \widehat{\gamma}_{j}^{2}}{\widetilde{\sigma_{0}^{2}+{\widetilde{\tau_{0}^{2}}}^{+} \sum_{j=0}^{I-i} w_{i j} \widehat{\gamma}_{j}^{2}}}
$$

Thus, the estimators of ultimate loss $C_{i j}$ and reserves $R_{i}$ are given by

$$
\begin{gathered}
\widehat{C}_{i J}^{\exp }=C_{i, I-i}+\left(1-\widehat{\beta}_{I-i}\right)\left[\widehat{Z}_{i}^{w} \bar{X}_{i}^{w}+\left(1-\widehat{Z}_{i}^{w}\right) \tilde{\nu}_{0}\right], \\
\widehat{R}_{i}^{\exp }=\left(1-\widehat{\beta}_{I-i}\right)\left[\widehat{Z}_{i}^{w} \bar{X}_{i}^{w}+\left(1-\widehat{Z}_{i}^{w}\right) \tilde{\nu}_{0}\right],
\end{gathered}
$$

for $i \in\{0,1,2, \ldots, I\}$. Correspondingly, the credibility estimator of the total reserve is

$$
\widehat{R}^{\exp }=\sum_{i=0}^{I}\left(1-\widehat{\beta}_{I-i}\right)\left[\widehat{Z}_{i}^{w} \bar{X}_{i}^{w}+\left(1-\widehat{Z}_{i}^{w}\right) \tilde{\nu}_{0}\right] .
$$

The estimator $\widehat{R}^{\exp }$ is called the empirical Bayesian estimator of total reserve. Obviously, the empirical Bayesian estimator does not depend on any unknown parameters and can be used directly in practice.

\section{Numerical Simulation}

In this paper, we give the Bühlmann-Straub credibility estimators of claim means, as well as the credibility estimator of the total reserve. In addition, the estimators of development factors and the structural parameters are discussed. Finally, the empirical Bayesian estimator of the reserve is obtained. In the following, we will use numerical simulation to verify two problems. First, we will compare and check the differences between the credibility estimates and Bayesian estimates of claim means; second, we will examine the difference between the empirical Bayesian estimates and the credibility estimates of reserves.
In the simulations, we take $w_{i j}=\left(1 / \gamma_{j^{2}}\right)$ and denote $Y_{i j}=\left(X_{i j} / \gamma_{j}\right)$. In addition, we assume that

$$
\left\{\theta_{i}=\frac{1}{\mu_{i}}, i=0,1,2, \ldots, I\right\},
$$

are i.i.d. random variable with $G a(\alpha, \beta)$ distribution, and the density function of $\theta_{i}$ is given by

$$
\pi(\theta)=\frac{\beta^{\alpha}}{\Gamma(\alpha)} \theta^{\alpha-1} \exp (-\beta \theta), \quad \theta>0 .
$$

Given $\mu_{i}$, the $\left\{Y_{i j}, j=0,1,2, \ldots\right\}$ are independent and distributed as follows:

$$
f\left(y \mid \theta_{i}\right)=\theta_{i} \exp \left(-\theta_{i} y\right), \quad y>0 .
$$

So we can easily get the following:

$$
\begin{aligned}
& v_{0}=\frac{\beta}{\alpha-1}, \\
& \tau_{0}^{2}=\frac{\beta^{2}}{(\alpha-1)^{2}(\alpha-2)}, \\
& \sigma_{0}^{2}=\frac{\beta^{2}}{(\alpha-1)(\alpha-2)}, \\
& Z_{i}=\frac{I-i+1}{I-i+1+\alpha-1} .
\end{aligned}
$$

In addition, we take $I=J=6, \alpha=2, \beta=1, \gamma_{j}=0.1$ for $j=0,1, \ldots, J$. Therefore, the posterior distribution of $\theta_{i}$ is given by

$$
\left(\theta_{i} \mid D_{I}\right) \sim G a\left(\alpha+\sum_{j=0}^{I-i} Y_{i j}, \beta+I-i+1\right) .
$$

In the simulation progress, we first generate a claim sequence $\left\{Y_{i, j}, i, j=0,1, \ldots, I\right\}$ based on the gamma distribution $G a(\alpha, \beta)$. In the second, the $X_{i, j}, C_{i, j}$ can be calculated for $i, j \in\{0,1, \ldots, I\}$. Thus, we get the following estimates:

(1) Chain ladder estimates (CL): $C_{i, J}^{C L}=C_{i, I-i}$ $f_{I-i} \ldots f_{J-1}$.

(2) B-F estimates (B-F): $C_{i, J}^{B-F}=C_{i, I-i}+\left(1-\beta_{I-i}\right) \widehat{\mu}_{i}$, where $\widehat{\mu}_{i}$ are the prior estimates of $\mu_{i}$ for $i \in\{0,1, \ldots, I\}$. Since there is no prior information, we directly take $\widehat{\mu}_{i}=\nu_{0}$ for $i \in\{0,1, \ldots, I\}$.

(3) Bayesian estimates (BE):

$$
\begin{aligned}
\widehat{C}_{i, J}^{*} & =C_{i, I-i}+\left(1-\beta_{I-i}\right) E\left(\mu_{i} \mid D_{I}\right) \\
& =C_{i, I-i}+\frac{\left(1-\beta_{I-i}\right)(\beta+I-i+1)}{\alpha+\sum_{j=0}^{I-i} Y_{i j}-1} .
\end{aligned}
$$

(4) Credibility estimates (CE):

$$
C_{i J}^{\text {cred }}=C_{i, I-i}+\left(1-\beta_{I-i}\right)\left(Z_{i} \bar{Y}_{i}+\left(1-Z_{i}\right) v_{0}\right) .
$$


Under 10000 simulations, the mean predictor error of $\mathrm{CL}, \mathrm{B}-\mathrm{F}, \mathrm{BE}$, and $\mathrm{CE}$ estimates is listed in the following tables.

The MSE in Table 1 is calculated under given parameters $\gamma_{j}, \beta_{j}, f_{j}$ and $\nu_{0}, \tau_{0}^{2}, \sigma_{0}^{2}$, while in Table 2 the MSE of empirical Bayesian version is calculated by inserting the estimator of the structure parameters with simulated data, and the prior estimators of B-F method are given by $\widehat{\mu}_{i}=v_{0}$ for $i \in\{0,1,2, \ldots, I\}$.

Obviously, from Table 1, the mean square error of the credibility estimates is smaller than that of CL estimates and $\mathrm{B}-\mathrm{F}$ estimates. Among these estimates, the CL estimates have the largest mean square error. The main reason is that $\mathrm{CL}$ estimates ignore the use of prior information. In conclusion, the simulation results show that the credibility estimation is a good choice within all these estimation methods in the random B-F model.

In Table 2, we estimate the parameters $\gamma_{j}, \beta_{j}, f_{j}$ and $v_{0}, \tau_{0}^{2}, \sigma_{0}^{2}$ by using simulated data $D_{I}$. In addition, the CL estimates, B-F estimates, and credibility estimates are derived correspondingly. At this time, the mean square error is increased due to the estimates of the structural parameters used in the empirical Bayesian setup. While the known prior estimates $\widehat{\mu}_{i}=v_{0}$ are still be used, the resulting credibility estimates have a slightly larger mean square error than the B-F estimator. However, the MSE of empirical Bayesian credibility estimator is still smaller than that of the CL estimates.

In the following, we apply this method to calculate the reserves in a practical example of an insurance company. The data come from Table 2.2 on page 20 of Alai et al.'s study. [11]. Based on the insurance claim data, we calculated the reserve estimates for the three methods including B-F method, chain ladder method, and credibility estimation. The results are listed as follows, where the prior estimates of the B-F method are taken as the same of Alai et al. [11], and the weights in credibility models are taken as $w_{i j}=\left(1 / \gamma_{j^{2}}\right)$ for $i \in\{0,1,2, \ldots, I\}$ and $j \in\{0,1,2, \ldots, J\}$ (Table 3 ).

From the table above, the total reserve obtained by the B-F method is larger than that of credibility estimates and CL estimates, mainly because the actuary obtains a larger value of the prior estimates $\mu_{i}$ for $i \in\{0,1,2, \ldots, I\}$. The reserve obtained by the credibility method is larger than that of CL estimates but is smaller than that of B-F estimates. Obviously, the reserve estimate obtained by the B-F method depends on the selection of the prior distribution, which is subjectively determined by the actuary, while the chain ladder method only uses sample information and ignores the use of prior information. The credibility estimates use both the sample information and prior information. However, the estimates derived by the credibility method do not depend on the specific distribution form of the prior distribution, which avoids the subjectivity of the selection of the prior distribution. Therefore, the credibility estimators are recommended to be used in the assessment of the liability reserve in insurance companies.

\section{Appendix}

Proof of Theorem 1. Let $Y_{i j}=\left(X_{i j} / \gamma_{j}\right)$. From Assumptions (1)-(3), we have

$$
\begin{gathered}
E\left(Y_{i, j} \mid \mu\right)=\mu_{i}, \\
\operatorname{Var}\left(Y_{i, j} \mid \mu\right)=\frac{\sigma^{2}\left(\mu_{i}\right)}{w_{i j} \gamma_{j}^{2}} .
\end{gathered}
$$

Denote $Y_{i}=\left(Y_{i 0}, \ldots, Y_{i, I-i}\right)^{T}$, then

$$
E\left(Y_{i}\right)=E\left[E\left(Y_{i} \mid \mu\right)\right]=\mu_{0} 1_{I-i+1},
$$

where $1_{I-i+1}=(1, \ldots, 1)^{T}$ indicates the $I-i+1$ vector with all entries 1 . By the double condition expectation formula of variance and covariance, we get

$$
\begin{aligned}
\operatorname{Cov}\left(\mu_{i}, Y_{i}\right) & =\operatorname{Cov}\left(\mu_{i}, E\left(Y_{i} \mid \mu\right)\right)=\tau_{0}^{2} 1_{I-i+1}^{T}, \\
\operatorname{Var}\left(Y_{i}\right) & =\operatorname{Var}\left(E\left(Y_{i} \mid \mu\right)\right)+E\left(\operatorname{Var}\left(Y_{i} \mid \mu\right)\right) . \\
& =\tau_{0}^{2} 1_{I-i+1} 1_{I-i+1}^{T}+\sigma_{0}^{2} G_{I-i+1},
\end{aligned}
$$

where

$$
G_{I-i+1}^{-1}=\left(\begin{array}{ccc}
w_{i 0} \gamma_{0}^{2} & & \\
& \ddots & \\
& & w_{i, I-i} \gamma_{I-i}^{2}
\end{array}\right)
$$

is the diagonal matrix. Thus, we have

$$
\begin{aligned}
\operatorname{Var}^{-1}\left(Y_{i}\right) & =\frac{1}{\sigma_{0}^{2}} G_{I-i+1}^{-1}-\frac{1}{\sigma_{0}^{2}} G_{I-i+1}^{-1} 1_{I-i+1}\left(\frac{1}{\tau_{0}^{2}}+1_{I-i+1}^{T} \frac{1}{\sigma_{0}^{2}} G_{I-i+1}^{-1} 1_{I-i+1}\right)^{-1} 1_{I-i+1}^{T} \frac{1}{\sigma_{0}^{2}} G_{I-i+1}^{-1} \\
& =\frac{1}{\sigma_{0}^{2}} G_{I-i+1}^{-1}-\frac{\tau_{0}^{2} G_{I-i+1}^{-1} 1_{I-i+1} 1_{I-i+1}^{T} G_{I-i+1}^{-1}}{\sigma_{0}^{2}\left(\sigma_{0}^{2}+\tau_{0}^{2} w_{i .}\right)},
\end{aligned}
$$


TABLe 1: The MSE of CL, B-F, BE, and CE under Bayesian setup.

\begin{tabular}{llccccr}
\hline$i$ & 0 & 1 & 2 & 3 & 4 & 5 \\
\hline CL & 0 & 9.3733 & 3.4652 & 1.8622 & 0.3549 & 1.3131 \\
B-F & 0 & 0.1441 & 0.2578 & 2.0759 & 0.8748 & 1.5095 \\
BE & 0 & 0.2077 & 0.4954 & 2.5547 & 1.5858 & 2.3768 \\
CE & 0 & 0.0993 & 0.1555 & 0.5230 & 0.5279 & 1.1630 \\
\hline
\end{tabular}

Table 2: The MSE of CL, B-F, BE, and CE under empirical Bayesian setup.

\begin{tabular}{llcccccc}
\hline$i$ & 0 & 1 & 2 & 3 & 4 & 5 \\
\hline CL & 0 & 25.6394 & 15.2681 & 2.9290 & 0.9422 & 0.6448 \\
B-F & 0 & 0.0524 & 0.1436 & 0.3754 & 0.7814 & 0.7540 & 1.9643 \\
Cred & 0 & 0.0774 & 0.2012 & 0.4290 & 0.7300 & 0.8169 \\
\hline
\end{tabular}

where $w_{i}=\sum_{j=0}^{I-i} w_{i j} \gamma_{j}^{2}$. According to the projection calculation formula of credibility theory (see Wen et al. [17]), we can derive

$$
\begin{aligned}
\widehat{\mu}_{i}^{B S} & =E\left(\mu_{i}\right)+\operatorname{Cov}\left(\mu_{i}, Y_{i}\right) \operatorname{Var}^{-1}\left(Y_{i}\right)\left(Y_{i}-E\left(Y_{i}\right)\right) \\
& =\mu_{0}+\tau_{0}^{2} 1_{I-i+1}^{T}\left(\frac{1}{\sigma_{0}^{2}} G_{I-i+1}^{-1}-\frac{\tau_{0}^{2} G_{I-i+1}^{-1} 1_{I-i+1} 1_{I-i+1}^{T} G_{I-i+1}^{-1}}{\sigma_{0}^{2}\left(\sigma_{0}^{2}+\tau_{0}^{2} w_{i} .\right)}\right)\left(Y_{i}-\mu_{0} 1_{I-i+1}\right) \\
& =\mu_{0}+\frac{\tau_{0}^{2}}{\sigma_{0}^{2}} 1_{I-i+1}^{T} G_{I-i+1}^{-1}\left(Y_{i}-\mu_{0} 1_{I-i+1}\right)-\frac{\tau_{0}^{4} 1_{I-i+1}^{T} G_{I-i+1}^{-1} 1_{I-i+1}}{\sigma_{0}^{2}\left(\sigma_{0}^{2}+\tau_{0}^{2} w_{i .}^{\prime}\right)} 1_{I-i+1}^{T} G_{I-i+1}^{-1}\left(Y_{i}-\mu_{0} 1_{I-i+1}\right) \\
& =\mu_{0}+\left(\frac{\tau_{0}^{2}}{\sigma_{0}^{2}}-\frac{\tau_{0}^{4} 1_{I-i+1}^{T} G_{I-i+1}^{-1} 1_{I-i+1}}{\sigma_{0}^{2}\left(\sigma_{0}^{2}+\tau_{0}^{2} w_{i .}\right)}\right) 1_{I-i+1}^{T} G_{I-i+1}^{-1}\left(Y_{i}-\mu_{0} 1_{I-i+1}\right) \\
& =\mu_{0}+\left(\frac{\tau_{0}^{2}}{\sigma_{0}^{2}}-\frac{\tau_{0}^{4} w_{i .}}{\sigma_{0}^{2}\left(\sigma_{0}^{2}+\tau_{0}^{2} w_{i \cdot}\right)}\right) \sum_{j=0}^{I-i} w_{i j} \gamma_{j}^{2}\left(Y_{i j}-\mu_{0}\right) \\
& =\left(1-Z_{i}^{w}\right) \mu_{0}+Z_{i}^{w} \sum_{j=0}^{I-i} \frac{w_{i j} \gamma_{j}^{2} Y_{i j}}{w_{i .}} . \\
& =\mu_{0}+\frac{\tau_{0}^{2}}{\sigma_{0}^{2}+\tau_{0}^{2} w_{i .}} \sum_{j=0}^{I-i} w_{i j} \gamma_{j}^{2}\left(Y_{i j}-\mu_{0}\right) \\
& =\mu_{0}+\frac{w_{i} \tau_{0}^{2}}{\sigma_{0}^{2}+\tau_{0}^{2} w_{i} \cdot} \sum_{j=0}^{I-i} \frac{w_{i j} \gamma_{j}^{2}\left(Y_{i j}-\mu_{0}\right)}{w_{i .}^{\prime}}
\end{aligned}
$$

Since

$$
\bar{X}_{i}^{w}=\frac{\sum_{j=0}^{I-i} w_{i j} \gamma_{j} X_{i j}}{\sum_{j=0}^{I-i} w_{i j} \gamma_{j}^{2}}=\frac{\sum_{j=0}^{I-i} w_{i j} \gamma_{j}^{2} Y_{i j}}{w_{i}}
$$

Proof of Proposition 1. According to models (3)-(9), it is easy to check that

$$
E\left(\bar{X}_{i}^{w} \mid \mu\right)=\frac{\sum_{j=0}^{I-i} w_{i j} \gamma_{j} E\left(X_{i j} \mid \mu\right)}{\sum_{j=0}^{I-i} w_{i j} \gamma_{j}^{2}}=\frac{\sum_{j=0}^{I-i} w_{i j} \gamma_{j} \mu_{i} \gamma_{j}}{\sum_{j=0}^{I-i} w_{i j} \gamma_{j}^{2}}=\mu_{i} .
$$


TABLE 3: Comparison of reserve estimates for the three methods.

\begin{tabular}{|c|c|c|c|c|c|c|c|}
\hline \multirow{2}{*}{ Policies $i$} & \multirow{2}{*}{$\begin{array}{c}\text { Prior estimates } \\
\widehat{\mu}_{i} \\
\end{array}$} & \multicolumn{4}{|c|}{ Estimates of ultimate loss } & \multicolumn{2}{|c|}{ Estimates of loss reserves } \\
\hline & & $\widehat{C}_{i J}^{B F}$ & $\widehat{C}_{i J}^{C L}$ & $\widehat{C}_{i J}^{C r e}$ & $\widehat{R}_{i}^{B F}$ & $\widehat{R}_{i}^{C L}$ & $\widehat{R}_{i}^{\text {Cre }}$ \\
\hline 0 & 11653101 & 11148124 & 11148124 & 11148124 & 0 & 0 & 0 \\
\hline 1 & 11367306 & 10664316 & 10663318 & 10661418 & 16124 & 13226.27 & 15126.29 \\
\hline 2 & 10962965 & 10662749 & 10662008 & 10661501 & 26998 & 25749.54 & 26257.45 \\
\hline 3 & 10616762 & 9761643 & 9758606 & 9759366 & 37575 & 35298.42 & 34538.47 \\
\hline 4 & 11044881 & 9882350 & 9872218 & 9886485 & 95434 & 99568.73 & 85301.62 \\
\hline 5 & 11480700 & 10113777 & 10092247 & 10086401 & 178024 & 150648.41 & 156494.25 \\
\hline 6 & 11413572 & 9623328 & 9568143 & 9575260 & 341305 & 293238.44 & 286092.48 \\
\hline 7 & 11126527 & 8830301 & 8705378 & 8733654 & 574089 & 477442.86 & 449163.52 \\
\hline 8 & 10986548 & 8967375 & 8691971 & 8791079 & 1318646 & 1142349.78 & 1043238.98 \\
\hline \multirow[t]{2}{*}{9} & 11618437 & 10443953 & 9626383 & 9805519 & 4768384 & 4129950.96 & 3950811.42 \\
\hline & & & & Total & 7356579 & 6367473.41 & 6047024.48 \\
\hline
\end{tabular}

Thus, we have $E\left(\bar{X}_{i}^{w}\right)=E\left[E\left(\bar{X}_{i}^{w} \mid \mu\right)\right]=\mu_{0}$. That is,

$$
E\left(\widehat{\mu}_{i}^{B S}\right)=Z_{i}^{w} E\left(\bar{X}_{i}^{w}\right)+\left(1-Z_{i}^{w}\right) \mu_{0}=\mu_{0}=E\left(\mu_{i}\right) .
$$
by

$$
\begin{aligned}
\operatorname{MSE}\left(\widehat{\mu}_{i}^{B S}\right) & =E\left[\left(Z_{i}^{w} \bar{X}_{i}^{w}+\left(1-Z_{i}^{w}\right) \mu_{0}-\mu_{i}\right)^{2}\right] \\
& =E\left[\left(Z_{i}^{w}\left(\bar{X}_{i}^{w}-\mu_{i}\right)+\left(1-Z_{i}^{w}\right)\left(\mu_{0}-\mu_{i}\right)\right)^{2}\right] \\
& =\left(Z_{i}^{w}\right)^{2} E\left[\left(\bar{X}_{i}^{w}-\mu_{i}\right)^{2}\right]+\left(1-Z_{i}^{w}\right)^{2} E\left[\left(\mu_{0}-\mu_{i}\right)^{2}\right]+2 Z_{i}^{w}\left(1-Z_{i}^{w}\right) E\left[\left(\bar{X}_{i}^{w}-\mu_{i}\right)\left(\mu_{0}-\mu_{i}\right)\right] \\
& =\left(Z_{i}^{w}\right)^{2} E\left[\operatorname{Var}\left(\bar{X}_{i}^{w} \mid \mu\right)\right]+\left(1-Z_{i}^{w}\right)^{2} \operatorname{Var}\left(\mu_{i}\right)+2 Z_{i}^{w}\left(1-Z_{i}^{w}\right) E\left[\left(\bar{X}_{i}^{w}-\mu_{i}\right)\left(\mu_{0}-\mu_{i}\right)\right] \\
& =\frac{\tau_{0}^{2} \sigma_{0}^{2}}{\sigma_{0}^{2}+\tau_{0}^{2} \sum_{j=0}^{I-i} w_{i j} \gamma_{j}^{2}} .
\end{aligned}
$$

The last "=" holds true due to the facts

$$
\begin{aligned}
E\left[\left(\bar{X}_{i}^{w}-\mu_{i}\right)\left(\mu_{0}-\mu_{i}\right)\right] & =E\left[E\left(\left(\bar{X}_{i}^{w}-\mu_{i}\right)\left(\mu_{0}-\mu_{i}\right) \mid \mu\right)\right] \\
& =E\left[\left(\mu_{0}-\mu_{i}\right) E\left(\left(\bar{X}_{i}^{w}-\mu_{i}\right) \mid \mu\right)\right]=0, \\
E\left[\operatorname{Var}\left(\bar{X}_{i}^{w} \mid \mu\right)\right] & =\frac{\sigma_{0}^{2}}{\sum_{j=0}^{I-i} w_{i j} \gamma_{j}^{2}}, \quad \operatorname{Var}\left(\mu_{i}\right)=\tau_{0}^{2} .
\end{aligned}
$$

Proof of Proposition 2. According to (14), we have obtained $\widehat{C}_{i J}^{*}=C_{i, I-i}+\left(1-\widehat{\beta}_{I-i}\right) E\left(\mu_{i} \mid D_{I}\right)$. From Theorem 3.1, we have

$$
\widehat{\mu}_{i}^{B S}=\widehat{E}\left(\mu_{i} \mid D_{I}\right)=Z_{i}^{w} \bar{X}_{i}^{w}+\left(1-Z_{i}^{w}\right) \mu_{0},
$$

and get (24). Finally, (25) and (26) are derived.
Proof of Theorem 2. Let $Y_{i j}=\left(X_{i j} / \gamma_{j}\right), b_{i j}=\alpha_{i j} \gamma_{j}$, the homogeneous linear function class of $\mu_{i}$ can be expressed as follows:

$$
L e(X)=\operatorname{Le}(Y)=\left\{\widehat{\mu}_{i}=\sum_{s=0}^{I} \sum_{t=0}^{I-s} b_{s t} Y_{s t}, \sum_{s=0}^{I} \sum_{t=0}^{I-s} b_{s t}=1\right\} .
$$

Thus, the optimization problem (28) is equivalent to

$$
\left\{\begin{array}{c}
\min _{\alpha, a_{s t} \in R} \mathrm{E}\left[\left(\mu_{i}-\sum_{s=0}^{I} \sum_{t=0}^{I-s} b_{s t} Y_{s t}\right)^{2}\right], \\
\sum_{s=0}^{I} \sum_{t=0}^{I-s} b_{s t}=1 .
\end{array}\right.
$$

Note that 


$$
\begin{gathered}
E\left(Y_{i, j} \mid \mu\right)=\mu_{i}, \\
\operatorname{Var}\left(Y_{i, j} \mid \mu\right)=\frac{\sigma^{2}\left(\mu_{i}\right)}{w_{i j} \gamma_{j}^{2}},
\end{gathered}
$$

which shows that it meets the conditions of standard Bühlmann-Straub models. Therefore, according to the procedures of solving the homogeneous estimation by Bühlmann and Straub, (29) is easily proved.

Proof of Proposition 3. For $i=0,1, \ldots, I-1$, we write

$$
S_{i}^{2}=\frac{1}{I-i} \sum_{j=0}^{I-i} w_{i j}\left(X_{i j}-\gamma_{j} \bar{X}_{i}^{w}\right)^{2}
$$

$$
\begin{aligned}
S_{i}^{2}= & \frac{1}{I-i} \sum_{j=0}^{I-i} w_{i j}\left(X_{i j}-\mu_{i} \gamma_{j}+\mu_{i} \gamma_{j}-\gamma_{j} \bar{X}_{i}^{w}\right)^{2} \\
= & \frac{1}{I-i}\left[\sum_{j=0}^{I-i} w_{i j}\left(X_{i j}-\mu_{i} \gamma_{j}\right)^{2}+\sum_{j=0}^{I-i} w_{i j}\left(\mu_{i} \gamma_{j}-\gamma_{j} \bar{X}_{i}^{w}\right)^{2}\right. \\
& \left.-\sum_{j=0}^{I-i} w_{i j}\left(X_{i j}-\mu_{i} \gamma_{j}\right)\left(\gamma_{j} \bar{X}_{i}^{w}-\mu_{i} \gamma_{j}\right)\right] .
\end{aligned}
$$

Suppose the $\left\{\gamma_{0}, \gamma_{1}, \ldots, \gamma_{J-1}\right\}$ are given constants, by double expectation formula, we get

Through the decomposition of $S_{i}^{2}$, we get

$$
\begin{aligned}
E\left(X_{i j}-\mu_{i} \gamma_{j}\right)^{2} & =E\left[E\left(\left(X_{i j}-\mu_{i} \gamma_{j}\right)^{2} \mid \mu\right)\right] \\
& =E\left[\operatorname{Var}\left(X_{i j} \mid \mu\right)\right]=\frac{\sigma_{0}^{2}}{w_{i j}}, \\
E\left(\mu_{i} \gamma_{j}-\gamma_{j} \bar{X}_{i}^{w}\right)^{2} & =E\left[\operatorname{Var}\left(\bar{X}_{i}^{w} \mid \mu\right)\right]=\frac{\gamma_{j}^{2}}{w_{i} .} \sigma_{0}^{2}, \\
E\left[\left(X_{i j}-\mu_{i} \gamma_{j}\right)\left(\gamma_{j} \bar{X}_{i}^{w}-\mu_{i} \gamma_{j}\right)\right] & =E\left[\operatorname{Cov}\left(X_{i j}, \bar{X}_{i}^{w} \mid \mu\right)\right]=\frac{\gamma_{j}^{2}}{w_{i} .} \sigma_{0}^{2} .
\end{aligned}
$$

Thus, we have

$$
\begin{aligned}
E\left(S_{i}^{2}\right)= & \frac{1}{I-i}\left[\sum_{j=0}^{I-i} w_{i j} E\left(X_{i j}-\mu_{i} \gamma_{j}\right)^{2}+\sum_{j=0}^{I-i} w_{i j} E\left(\mu_{i} \gamma_{j}-\gamma_{j} \bar{X}_{i}^{w}\right)^{2}\right. \\
& \left.-2 \sum_{j=0}^{I-i} w_{i j} E\left(X_{i j}-\mu_{i} \gamma_{j}\right)\left(\gamma_{j} \bar{X}_{i}^{w}-\mu_{i} \gamma_{j}\right)\right] \\
= & \frac{1}{I-i}\left[(I-i+1) \sigma_{0}^{2}-\sigma_{0}^{2}\right] \\
= & \sigma_{0}^{2} .
\end{aligned}
$$

Therefore, the expectation of $\widehat{\sigma_{0}^{2}}$ is given by

$$
E\left(\widehat{\sigma_{0}^{2}}\right)=E\left(\frac{1}{I} \sum_{i=0}^{I-1} S_{i}^{2}\right)=\sigma_{0}^{2}
$$

Proof of Proposition 4. For the convenience of notations, let

$$
T=\sum_{i=0}^{I-1} \frac{w_{i \cdot}}{w_{. .}}\left(\bar{X}_{i}^{w}-\overline{\bar{X}}^{w}\right)^{2} .
$$

Then, the $\widehat{\tau_{0}^{2}}$ can be rewritten as

$$
\widehat{\tau_{0}^{2}}=\frac{\left(w_{. .}\right)^{2}}{\left(w_{. .}\right)^{2}-\sum_{i=0}^{I-1}\left(w_{i .}\right)^{2}}\left(T-\frac{I}{w_{. .}} \widehat{\sigma_{0}^{2}}\right)
$$

Note that

$$
E\left(\bar{X}_{i}{ }^{w}-\overline{\bar{X}}^{w}\right)^{2}=\operatorname{Var}\left(\bar{X}_{i}^{w}\right)+\operatorname{Var}\left(\overline{\bar{X}}^{w}\right)-2 \operatorname{Cov}\left(\bar{X}_{i}^{w}, \overline{\bar{X}}^{w}\right)
$$

Then, three terms of the right side of the above equation are given by 


$$
\begin{aligned}
\operatorname{Var}\left(\bar{X}_{i}^{w}\right) & =E\left[\operatorname{Var}\left(\bar{X}_{i}^{w} \mid \mu\right)\right]+\operatorname{Var}\left[E\left(\bar{X}_{i}^{w} \mid \mu\right)\right]=\frac{\sigma_{0}^{2}}{w_{i .}}+\tau_{0}^{2}, \\
\operatorname{Var}\left(\overline{\bar{X}}^{w}\right) & =\frac{1}{\left(w_{. .}\right)^{2}} \sum_{i=0}^{I-1}\left(w_{i .}\right)^{2}, \\
\operatorname{Var}\left(\bar{X}_{i}^{w}\right) & =\frac{1}{\left(w_{. .}\right)^{2}} \sum_{i=0}^{I-1}\left(w_{i .}\right)^{2}\left(\frac{\sigma_{0}^{2}}{w_{i .}^{\prime}}+\tau_{0}^{2}\right)=\frac{\sigma_{0}^{2}}{w_{. .}}+\frac{\sum_{i=0}^{I-1}\left(w_{i .}\right)^{2}}{\left(w_{. .}\right)^{2}} \tau_{0}^{2}, \\
\operatorname{Cov}\left(\bar{X}_{i}^{w}, \overline{\bar{X}}^{w}\right) & =\frac{w_{i .}}{w_{. .}}, \\
\operatorname{Var}\left(\bar{X}_{i}^{w}\right) & =\frac{w_{i .}}{w_{. .}}\left(\frac{\sigma_{0}^{2}}{w_{i .}}+\tau_{0}^{2}\right)=\frac{\sigma_{0}^{2}}{w_{. .}}+\frac{w_{i .}}{w_{. .}} \tau_{0}^{2},
\end{aligned}
$$

respectively. By inserting them into (A.23), we get

$$
E\left(\bar{X}_{i}^{w}-\overline{\bar{X}}^{w}\right)^{2}=\frac{\sigma_{0}^{2}}{w_{i .}}+\tau_{0}^{2}-\frac{\sigma_{0}^{2}}{w_{. .}}+\frac{\sum_{i=0}^{I-1}\left(w_{i .}\right)^{2}}{\left(w_{i .}\right)^{2}} \tau_{0}^{2}-2 \frac{w_{i .}}{w_{. .}} \tau_{0}^{2} .
$$

Hence, we have

$$
\begin{aligned}
E(T) & =\sum_{i=0}^{I-1} \frac{w_{i .}}{w_{. .}} E\left(\bar{X}_{i}^{w}-\overline{\bar{X}}^{w}\right)^{2} \\
& =\sum_{i=0}^{I-1} \frac{w_{i \cdot}}{w_{. .}}\left[\frac{\sigma_{0}^{2}}{w_{i .}}+\tau_{0}^{2}-\frac{\sigma_{0}^{2}}{w_{.}}+\frac{\sum_{i=0}^{I-1}\left(w_{i .}\right)^{2}}{\left(w_{. .}\right)^{2}} \tau_{0}^{2}-2 \frac{w_{i .}}{w_{. .}} \tau_{0}^{2}\right] \\
& =\frac{I}{w_{. .}} \sigma_{0}^{2}+\left(1-\frac{\sum_{i=0}^{I-1}\left(w_{i .}\right)^{2}}{\left(w_{. .}\right)^{2}}\right) \tau_{0}^{2} .
\end{aligned}
$$

Therefore,

$$
\begin{aligned}
E\left(\widehat{\tau_{0}^{2}}\right)= & \frac{\left(w_{. .}\right)^{2}}{\left(w_{. .}\right)^{2}-\sum_{i=0}^{I-1}\left(w_{i .}\right)^{2}}\left[E(T)-\frac{I}{w_{. .}} E\left(\widehat{\sigma_{0}^{2}}\right)\right] \\
= & \frac{\left(w_{. .}\right)^{2}}{\left(w_{. .}\right)^{2}-\sum_{i=0}^{I-1}\left(w_{i .}\right)^{2}} \\
& \cdot\left[\frac{I}{w_{. .}} \sigma_{0}^{2}+\left(1-\frac{\sum_{i=0}^{I-1}\left(w_{i .}\right)^{2}}{\left(w_{. .}\right)^{2}}\right) \tau_{0}^{2}-\frac{I}{w_{. .}} E\left(\widehat{\sigma_{0}^{2}}\right)\right]=\tau_{0}^{2} .
\end{aligned}
$$

This shows that the $\widehat{\tau_{0}^{2}}$ is an unbiased estimator of $\tau_{0}^{2}$.

\section{Data Availability}

The data used to support the findings of this study are available from the corresponding author upon request.

\section{Conflicts of Interest}

The authors declare that they have no conflicts of interest.

\section{Acknowledgments}

The research was supported by the National Natural Science Foundation of China (71761019 and 11561026) and Science and Technology Project of Education Department of Jiangxi Province (GJJ180267).

\section{References}

[1] T. Mack, "Distribution-free calculation of the standard error of chain ladder reserve estimates," ASTIN Bulletin, vol. 23, no. 2, pp. 213-225, 1993.

[2] R. L. Bornhuetter and R. E. Ferguson, "The actuary and IBNR," Proceedings of the Casualty Actuarial Society Casualty Actuarial Society, vol. LIX, pp. 181-195, 1972.

[3] G. Quarg and T. Mack, "Munich chain ladder," Blatter DGVFM, vol. 116, pp. 597-630, Springer, Berlin, Germany, 2004.

[4] W. Hurlimann, "Approximate bounds for the IBNR claims reserves based on the bivariate chain ladder models," Belgian Acutarial Bulletin, vol. 5, no. 1, pp. 46-51, 2005.

[5] M. Pesta and S. Hudecová, "Asymptotic consistency and inconsistency of the chain ladder," Insurance: Mathematics and Economics, vol. 51, no. 2, pp. 472-479, 2012.

[6] U. Riegel, "A bifurcation approach for attritional and large losses in chain ladder calculations," ASTIN Bulletin, vol. 44, no. 1, pp. 127-172, 2014.

[7] C. Leonardo, P. Adrian, and A. Rodrigo, "Modeling and predicting IBNR reserve: extended chain ladder and heteroscedastic regression analysis," Journal of Applied Statistics, vol. 43, no. 5, pp. 847-870, 2016.

[8] A. Boratyńska, "Robust Bayesian estimation and prediction of reserves in exponential model with quadratic variance function," Insurance: Mathematics and Economics, vol. 76, pp. 135-140, 2017.

[9] F. Wahl, M. Lindholm, and R. Verrall, "The collective reserving model," Insurance: Mathematics and Economics, vol. 87, pp. 34-50, 2019.

[10] R. J. Verrall, "A Bayesian generalized linear model for the Bornhuetter-Ferguson method of claims reserving," North American Actuarial Journal, vol. 8, no. 3, pp. 67-89, 2004.

[11] D. H. Alai, M. Merz, and M. V. Wüthrich, "Mean square error of prediction in the bornhuetter-ferguson claims reserving method," Annals of Actuarial Science, vol. 4, no. 1, pp. 7-31, 2009. 
[12] A. Saluza, A. Gisler, and M. V. Wüthrich, "Development pattern and prediction error for the stochastic bornhuetterferguson claims reserving method," ASTIN Bulletin, vol. 41, no. 2, pp. 279-313, 2011.

[13] H. Jochen and T. Anne, "The fuzzy bornhuetter-ferguson method: an approach with fuzzy numbers," Annals of Actuarial Science, vol. 10, no. 2, pp. 303-321, 2016.

[14] D. Gogol, "Using expected loss ratios in reserving," Insurance: Mathematics and Economics, vol. 12, no. 3, pp. 297-299, 1993.

[15] H. Bühlmann, "Experience rating and credibility," Astin Bulletin, vol. 4, no. 3, pp. 199-207, 1967.

[16] H. Bühlmann and A. Gisler, A Course in Credibility Theory and its Applications, Springer, Berlin, Germany, 2005.

[17] L. Wen, X. Wu, and X. Zhou, "The credibility premiums for models with dependence induced by common effects," Insurance: Mathematics and Economics, vol. 44, no. 1, pp. 19-25, 2009.

[18] L. Wen, J. Yu, G. Mei, and Y. Zhang, "The credibility premiums based on estimated moment-generating function," Communications in Statistics-Theory and Methods, vol. 46, no. 3, pp. 1090-1106, 2017.

[19] C. C.-L. Tsai and A. D. Wu, "Incorporating hierarchical credibility theory into modelling of multi-country mortality rates," Insurance: Mathematics and Economics, vol. 91, pp. 37-54, 2020.

[20] G. Benktander, "An approach to credibility in calculating IBNR for casualty excess reinsurance," The Actuarial Review, vol. 3, no. 2, p. 7, 1976.

[21] E. Hovinen, Additive and Continuous IBNR, ASTIN Colloquium, Loen, Norway, 1981.

[22] O. Hesselager and T. Witting, "A credibility model with random fluctuations in delay probabilities for the prediction of IBNR claims," ASTIN Bulletin, vol. 18, no. 1, pp. 79-90, 1988.

[23] F. Vylder De, "Estimation of IBNR claims by credibility theory," Insurance Mathematics and Economics, vol. 1, pp. 35-40, 1982.

[24] A. Gisler and M. V. Wüthrich, "Credibility for the chain ladder reserving method," ASTIN Bulletin, vol. 38, no. 2, pp. 565-600, 2008.

[25] G. Taylor, "A Cape Cod model for the exponential dispersion family," Insurance: Mathematics and Economics, vol. 85, pp. 126-137, 2019. 